\title{
Generation of low-threshold optofluidic lasers in a stable Fabry-Pérot microcavity
}

Tingting Zhang, Chunhua Zhou, WenjieWang ${ }^{*}$ and Jingdong Chen

Key Lab of Advanced Transducers and Intelligent Control System of Ministry of Education, Taiyuan University of Technology, 79 Yingze Street, Taiyuan 030024, P. R. China

"wangwenjie@tyut.edu.cn

\begin{abstract}
We present the generation of optofluidic lasers in a Fabry-Pérot (FP) microcavity with low pumping threshold. The FP microcavity is constructed with one micro-concave mirror and another planar mirror facing each other to form a stable cavity. The two mirrors are coated with dielectric layers with reflectivity $\geq 99.9 \%$. Laser behaviors are tested with gain medium, Rhodamine 6G (R6G), dissolved in ethanol and ion water, respectively; a lowest pumping threshold as small as $0.13 \mu \mathrm{J} / \mathrm{mm}^{2}$ is achieved in the ethanol solution, corresponding to a theoretically calculated quality value $\mathrm{Q}_{0}$ about $4 \times 10^{5}$. Meanwhile experimental results demonstrates that the lowest concentration of dye medium that can emit laser signal in the FP microcavity is $3 \mu \mathrm{M}$.
\end{abstract}

Key word: Optofluidic laser, Fabry- Pérot microcavity, Dye laser, Nonlinear optics 


\section{Introduction}

Through the integration of microfluidics and optics, the field of optofluidics has emerged, which has shown large potential for optical devices and has already enabled a broad optical toolbox for lab-on-a-chip system[1]. Optofluidic dye lasers, as one of the promising technology, are of particular interest because of their dynamically tunable emission spectrum[2], various integrations with microfluidics, and great potential of applications in bio- and chemical sensing[3]. Various optical cavities have been explored for laser resonating, such as Fabry-Pérot (FP) cavity[4-6], distributed feedback grating[7-9], optical ring resonator[10-12], droplet[13, 14] and 3D optofluidic waveguide combined with FP cavity[15]. Among them, FP cavity has good compatibility with microfluidics, which can function both as sensing cavity and fluidic channel. Moreover, FP cavity provides bulk interaction of electromagnetic fields with the gain medium in the whole space, which can, on one side, improve the detection sensitivity, such as by eliminating the background fluorescence signal resulting from the un-interacted gain medium with the evanescent filed in optical ring resonator, meanwhile improve the utilization efficiency of solution and decrease the detection volume of analyte; on the other hand, enable the whole body detection, which is particularly useful for cell detection[16]. However, FP cavity usually suffers from tilt loss with light oscillating at a divergence angle in the cavity and easily escaping out; as for fiber-based FP cavity, precise alignment process is hard to be realized in microfluidics, thus resulting in low cavity quality factor (Q) and low laser threshold in optofluidic application[5, 17, 18]. Substantial efforts have been made in micro-fabrication of curved cavity with essential space confinement of light, such as wet etching method that can fabricate cylindrical and spherical concave surfaces in silicon[19-21]. $\mathrm{CO}_{2}$ laser ablation is another useful technique to create micro-concave surface at the tip of fibers or on planar fused silica substrates with variable radius of curvature and surface roughness as small as $0.2 \mathrm{~nm}$, as reported[22-24].

Here, we report the generation of low-threshold optofluidic laser in a FP microcavity consisting of one planar and one concave mirror. The concave mirror is fabricated using the method of $\mathrm{CO}_{2}$ laser ablation and subsequent dielectric coating. Gain medium, Rhodamine $6 \mathrm{G}$ (R6G), is dissolved in different solutions, with a lowest pumping threshold as small as 0.13 $\mu \mathrm{J} / \mathrm{mm}^{2}$ being obtained in the ethanol solution. The corresponding quality value ( $\mathrm{Q}$ factor) of the microcavity is estimated to be approximately $4 \times 10^{5}$. The lowest concentration of the dye medium that can emit laser signal in the FP microcavity is $3 \mu \mathrm{M}$.

\section{Experimental setup}

The concave micro-structure was fabricated on the optically polished surface of a fused silica substrate using an RF-pumped $\mathrm{CO}_{2}$ laser[24]. A typical concave structure is shown in Fig. 1(a) with inset showing the 3D SEM (scanning electron microscopy) image and curve 1 representing the $1 \mathrm{D}$ concave profile. The $1 \mathrm{D}$ concave profile can be approximated using a 
Gaussian function (curve 2) with parameters $t$ (depth), $d$ (effective width where the radius of curvature along the profile is positive), and $r$ (the minimum radius of curvature of the concave profile). Curve 3 depicts the distribution of radius of curvature along the concave profile with a minimum value $r$ located at the bottom of the concave structure. Thus concave profile near the bottom can be approximated using a circular distribution with radius $r$, as shown in curve 4 ; correspondingly the 3D concave structure can be approximately treated as an spherical structure with radius $r$ along the bottom of the structure. In the experiment, typical parameter range of the micro-concave structures are $t(8 \sim 13 \mu \mathrm{m}), d(85 \sim 105 \mu \mathrm{m})$, and $r(150 \sim 400 \mu \mathrm{m})$. An atomic force microscopy was used to investigate the surface roughness of the concave structure, where an average surface roughness rms (root mean square) of $2.5 \mathrm{~nm}$ over an area of $4 \mu \mathrm{m}^{2}$ can be achieved, indicating that low roughness surface is obtained using $\mathrm{CO}_{2}$ laser ablation in our experiment. Subsequent dielectric coating with maximum reflectivity $\geq 99.9 \%$ at center wavelength $600 \mathrm{~nm}$ was layered on the fused silica substrates with concave structures and those with planar surface only; such high reflectivity of the mirrors guarantees high finesse of the FP microcavity.

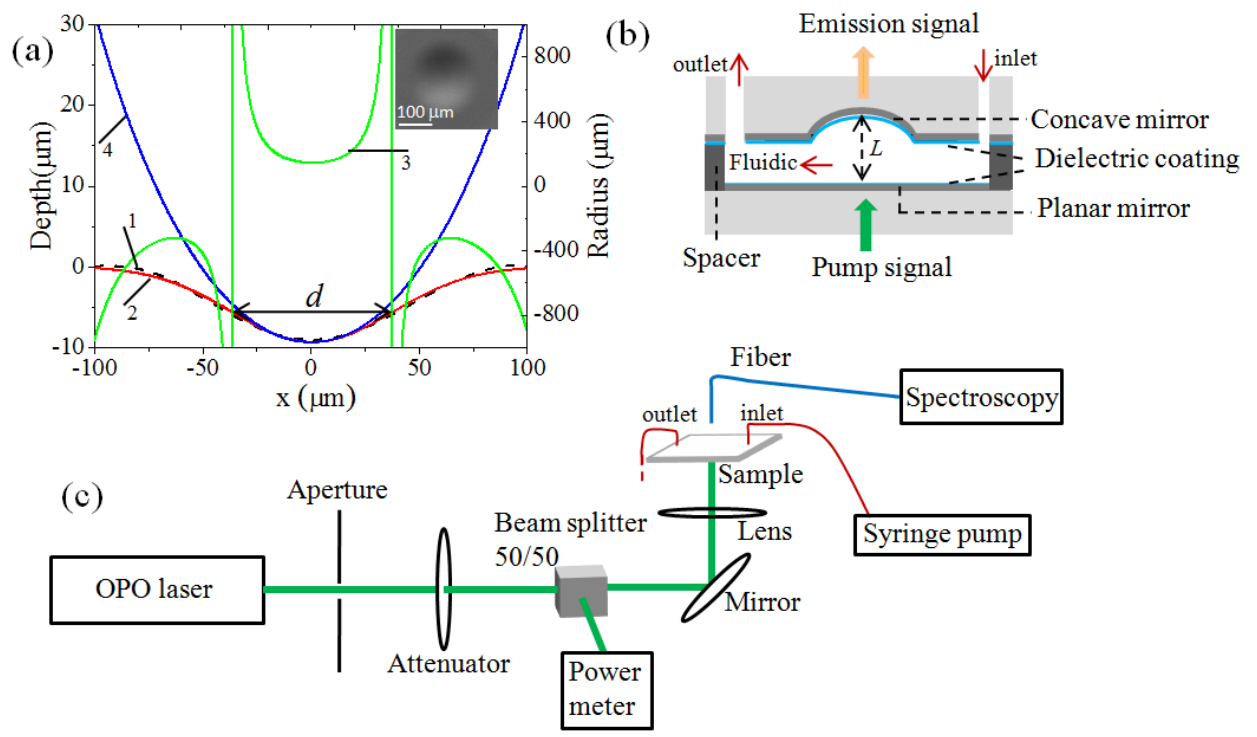

Fig 1. (a) A concave micro-structure. Inset: 3D SEM (scanning electron microscopy) image; curve 1: experimentally measured 1D concave profile; curve 2: Gaussian fit of the concave profile; curve 3: radius of curvature of the concave profile; curve 4: circular fit of the concave profile with radius r. $d$ is the effective width where the radius of curvature along the concave profile is positive. (b) Schematic depict of the FP microcavity and microfluidic channel. $L$ is the cavity length. (c) Experimental setup for optofluidic laser generation in the FP microcavity.

The FP microcavity is constructed with one planar and one concave mirror facing each other using a copper of thickness $30 \mu \mathrm{m}$ as the spacer, as schematically illustrated in Fig. 1(b). $L$ is the cavity length which equals to the thickness of the spacer plus the depth of the concave 
mirror. As discussed previously, the concave mirror can be approximately treated as a spherical mirror with radius $r$ near the bottom, thus when the cavity length $L$ is set to be less than the minimum radius $r$ of the concave mirror, the titling loss of the cavity will be minimized and a stable planar-to-concave laser cavity can be constructed, resulting in a stable laser mode in all dimensions[25]. Based on the above properties of the mirrors and the FP cavity, high Q factor of the microcavity and low pumping threshold of optofluidic lasers are expected.

A fluidic channel can be formed simultaneously, as shown in Fig. 1(b), where the solution of gain medium injected by a syringe pump can flow through at a velocity of $3 \mathrm{~mm} / \mathrm{s}$. The experimental setup for laser generation in the microcavity is depict in Fig. 1(c). A pulsed laser (532 nm wavelength, $5 \mathrm{~ns}$ pulse width, $20 \mathrm{~Hz}$ repetition rate) is normally incident into the FP microcavity through the planar mirror with a focal spot size of $100 \mu \mathrm{m}$. The pump energy can be varied using a neutral density attenuator. Dye emission signal in the cavity is collected from the end of the concave mirror with an optical fiber and then sent to a spectrometer (Horiba 320 ).

\section{Results and discussion}
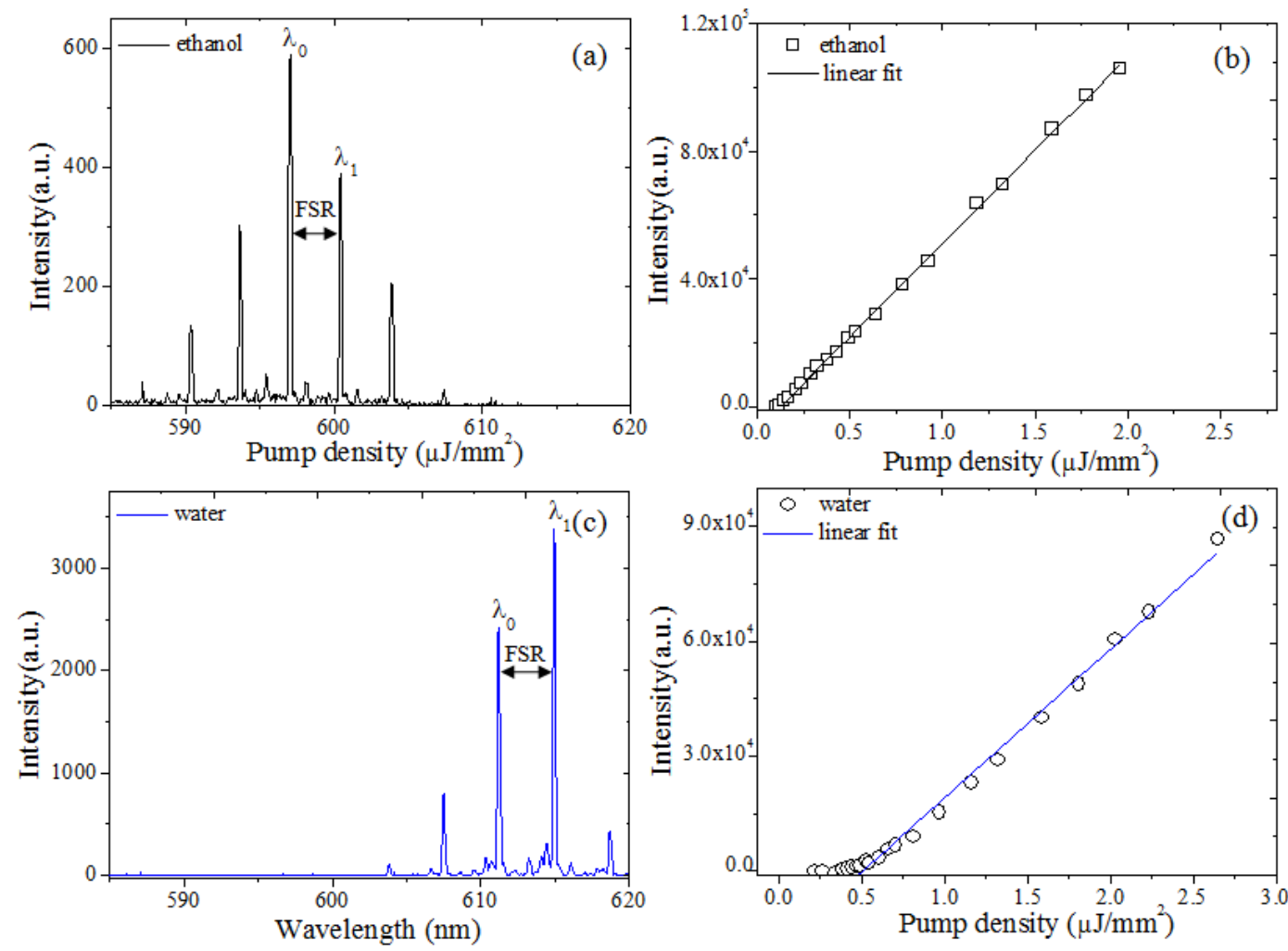

Fig 2. Laser spectrum distribution with R6G dissolved in ethanol (a) and ion water (c), respectively, in a FP microcavity with the corresponding pumping density $0.17 \mu \mathrm{J} / \mathrm{mm}^{2}$ and $1.3 \mu \mathrm{J} / \mathrm{mm}^{2}$, and FSR 3.37 $\mathrm{nm}$ and $3.72 \mathrm{~nm}$. (b) and (d) plot the spectrally integrated emission intensity of the resonance laser signals from $580 \mathrm{~nm}$ to $630 \mathrm{~nm}$ as a function of the pump density, with the gain medium dissolved in 
ethanol and ion water, respectively; correspondingly, the linear fit shows a pumping threshold of 0.13 $\mu \mathrm{J} / \mathrm{mm}^{2}$ and $0.45 \mu \mathrm{J} / \mathrm{mm}^{2}$, respectively.

First, the laser behavior of R6G dissolved in ethanol with a concentration of $1 \mathrm{mM}$ was tested. Fig. 2(a) shows the laser emission spectrum of the FP cavity at pump density of $0.17 \mu \mathrm{J} / \mathrm{mm}^{2}$ with resonance peaks centered at $\lambda_{0}=597 \mathrm{~nm}$. The free spectrum range $(F S R)$ of the cavity in wavelength is $F S R=\Delta \lambda=\lambda_{1}-\lambda_{0}=3.37 \mathrm{~nm}$, corresponding to a cavity length $L=\lambda_{1} \lambda_{0} /(2 n \Delta \lambda)=39 \mu \mathrm{m}$, close to the nominal spacer's thickness $(30 \mu \mathrm{m})$ plus the concave depth $(9.2 \mu \mathrm{m})$, provided that the refractive index of the ethanol solution is $n=1.362$. Spectrally integrated emission intensity of resonance signals from $580 \mathrm{~nm}$ to $630 \mathrm{~nm}$ as a function of the pump density is displayed in Fig. 2(b), where the corresponding linear fit shows that the lasing threshold in the FP cavity is approximately $0.13 \mu \mathrm{J} / \mathrm{mm}^{2}$. The threshold value is actually pretty low, to our best of acknowledge, among the FP cavities developed now, and even is comparable with that of the optofluidic optical ring resonators that usually exhibit very high $\mathrm{Q}$ value $\left(>10^{6}\right)$ and low laser threshold $\left(\sim 0.09 \mu \mathrm{J} / \mathrm{mm}^{2}\right)[10]$.

The lasing behavior of the cavity system for a given dye concentration can be described with a parameter $\gamma(\lambda)$, the minimum fraction of the excited molecules,

$$
\gamma(\lambda)=\frac{\sigma_{a}\left(\lambda_{L}\right)}{\sigma_{e}\left(\lambda_{L}\right)}\left(1+\frac{Q_{a b s}}{Q_{0}}\right)
$$

where $\sigma_{a}$ and $\sigma_{e}$ represent the absorption and emission cross section, respectively; $\lambda_{L}$ is the emission wavelength; $Q_{a b s}$ is the $\mathrm{Q}$ factor related to the dye absorption, i.e., $Q_{a b s}=2 \pi n /\left(\lambda_{L} n_{T} \sigma_{a}\left(\lambda_{L}\right)\right)$, where $n$ and $n_{T}$ denotes the refractive index of the solvent and the total concentration of dye molecules, respectively. $Q_{0}$ is quality factor of the empty cavity when the cavity is filled with solvent in the absence of dye, which is determined by the geometrical loss of the cavity and the absorption loss of the solvent. According to the three-level model at steady state, $\gamma$ is directly related to the pump threshold $I_{t h}$. After a straightforward derivation, $I_{t h}$ is obtained as

$$
I_{\text {th }}=\frac{h c \Delta t}{q \tau_{r a d} \sigma_{a}\left(\lambda_{p}\right) \lambda_{p}} \times \frac{B(1+C)}{1-B C}
$$

where $\Delta t(5 \mathrm{~ns}), q(0.9), \tau_{\text {rad }}(5 \mathrm{~ns})$, and $\lambda_{p}(532 \mathrm{~nm})$ represent the pulse width, quantum yield, radiative lifetime and pump wavelength, respectively, in our experiment; $B=\sigma_{a}\left(\lambda_{L}\right) / \sigma_{e}\left(\lambda_{L}\right)$ and $C=Q_{a b s} / Q_{0}$. For laser emission in the FP cavity with R6G dissolved in ethanol solvent, if we take $\lambda_{L}=597 \mathrm{~nm}, n=1.362, \sigma_{a}\left(\lambda_{L}\right)=1.5 \times 10^{-19} \mathrm{~cm}^{2}$, $\sigma_{e}\left(\lambda_{L}\right)=10^{-16} \mathrm{~cm}^{2}$, and laser threshold $I_{\text {th }}=0.13 \mu \mathrm{J} / \mathrm{mm}^{2}$, then the estimated $Q_{0}$ is $4 \times 10^{5}$.

Then, we test the laser performance of the same cavity with R6G dissolved in the solvent of ion water. The concentration of R6G is the same as that in ethanol solvent. One typical spectrum with the pumping density at $1.3 \mu \mathrm{J} / \mathrm{mm}^{2}$ is shown in Fig. 2(c) where the lasing wavelength is centered at $\lambda_{0}=615 \mathrm{~nm}$ with $F S R=3.72 \mathrm{~nm}$. The results indicate that a red-shift of wavelength about $18 \mathrm{~nm}$ occurs in the case of ion water solution. Accordingly, the 
dependence of the integrated spectrum intensity on the pump density is plotted in Fig. 2(d), with a corresponding linear fit showing a laser threshold of $0.45 \mu \mathrm{J} / \mathrm{mm}^{2}$. Those results indicate that a higher loss happens when we exchange the solvent from ethanol to ion water, thus an more intense pump density is required for lasing; meanwhile the lasing wavelength will red-shift to the place where the absorption loss of the dye molecular is smaller. The higher loss will result in a smaller quality factor $\mathrm{Q}_{0}$ of the microcavity, which is may due to a relatively larger absorption loss of the water solvent and smaller refractive index of ion water. If we take $\lambda_{L}=615 \mathrm{~nm}, n=1.3, \sigma_{a}\left(\lambda_{L}\right)=0.5 \times 10^{-19} \mathrm{~cm}^{2}, \sigma_{e}\left(\lambda_{L}\right)=0.7 \times 10^{-16} \mathrm{~cm}^{2}$, then the estimated quality factor $Q_{0}$ is $1.3 \times 10^{5}$.

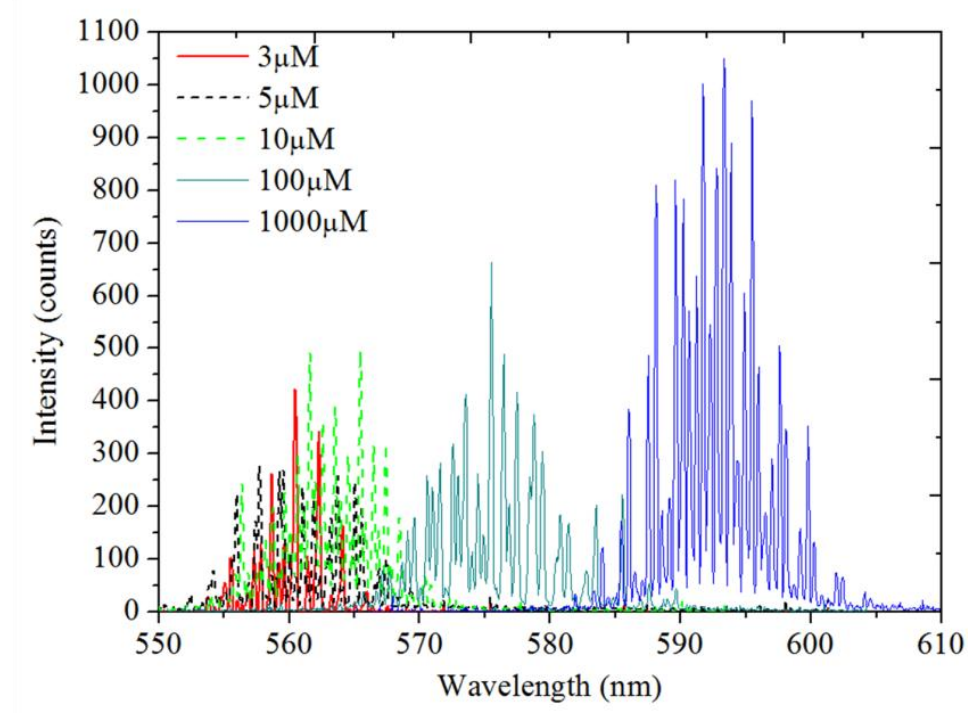

Fig 3. Intensity dependence of the resonant signals on the wavelengths in ethanol solutions dissolved with different concentrations of R6G as $1 \mathrm{mM}, 0.1 \mathrm{mM}, 0.01 \mathrm{mM}, 5 \mu \mathrm{M}$, and $3 \mu \mathrm{M}$, respectively.

High Q-value resonance cavities have wide applications in sensing, such as optofluidic lasing in biological sensing. To explore the minimum concentration of gain medium in solution that can generate laser emission signal in the FP microcavity, we measured the laser spectrum of the FP cavity with R6G dissolved in ethanol at different concentrations $1 \mathrm{mM}$, $0.1 \mathrm{mM}, 0.01 \mathrm{mM}, 5 \mu \mathrm{M}, 3 \mu \mathrm{M}$, as depict in Fig. 3. With the decrease of dye concentrations, i.e. an increase of $Q_{a b s}$, the central wavelength of the emission from $597 \mathrm{~nm}$ shifts to 560 $\mathrm{nm}$, which is the wavelength that corresponds to the maximum fluorescence emission $\left(\sigma_{e}\right)$ of R6G. As discussed in equations (1) and (2), when $Q_{a b s} \gg Q_{0}$, the laser threshold and emission wavelength is mainly determined by $1 / \sigma_{e}$, thus the laser emission will shift gradually to the shorter wavelength. When we reduce the concentration of R6G in ethanol to 2 $\mu \mathrm{M}$, the lasing oscillation cannot be motivated, which means that the gain obtained from the dye medium in the FP cavity cannot compensate for the cavity loss any more. This results is similar to that in the optofluidic ring resonator where the lowest detectable concentration of dye molecular is $2 \mu \mathrm{M}[10]$. If much lower concentration of gain medium excepted to be detected, higher $\mathrm{Q}$ value of optofluidic cavities has to be pursued. 


\section{Conclusion}

In summary, we have demonstrated the laser generation with low pumping threshold in an FP microcavity, which is formed by one planar and one concave dielectric-coated mirror. A threshold value as low as $0.13 \mu \mathrm{J} / \mathrm{mm}^{2}$ has been observed experimentally, corresponding to an estimated quality factor $Q_{0}$ of the microcavity $4 \times 10^{5}$. The lowest concentration of dye molecular that can generate laser signal in the FP microcavity is about $3 \mu \mathrm{M}$, comparable with the results in optofluidic ring resonator.

\section{Acknowledgement}

This work was supported by the National Science Foundation of China (Grant No. 61471254, 11204206, and 11304219), Research Project (Grant No. 2013-037, 201601D011010) Supported by Shanxi Scholarship Council of China.

\section{Reference}

[1] D. Psaltis, S.R. Quake, C. Yang, Developing optofluidic technology through the fusion of microfluidics and optics, Nature 442(7101) (2006) 381-6.

[2] J.C. Galas, J. Torres, M. Belotti, Q. Kou, Y. Chen, Microfluidic tunable dye laser with integrated mixer and ring resonator, Appl. Phys. Lett. 86(26) (2005) 264101.

[3] X. Fan, S.H. Yun, The potential of optofluidic biolasers, Nature Methods 11(2) (2014) 141-147.

[4] B. Helbo, A. Kristensen, A. Menon, A micro-cavity fluidic dye laser, J. Micromech. Microeng. 13 (2003) 307-311.

[5] Q. Kou, I. Yesilyurt, Y. Chen, Collinear dual-color laser emission from a microfluidic dye laser, Appl. Phys. Lett. 88(9) (2006) 091101.

[6] P. Liu, H. Huang, T. Cao, Z. Tang, X. Liu, Z. Qi, M. Ren, H. Wu, An optofluidics biosensor consisted of high-finesse Fabry-Pérot resonator and micro-fluidic channel, Appl. Phys. Lett. 100(23) (2012) 233705.

[7] M. Gersborg-Hansen, A. Kristensen, Optofluidic third order distributed feedback dye laser, Appl. Phys. Lett. 89(10) (2006) 103518.

[8] Z. Li, Z. Zhang, T. Emery, A. Scherer, D. Psaltis, Single mode optofluidic distributed feedback dye laser, Opt. Express 14 (2006) 696-701.

[9] W. Song, A.E. Vasdekis, Z. Li, D. Psaltis, Low-order distributed feedback optofluidic dye laser with reduced threshold, Appl. Phys. Lett. 94(5) (2009) 051117.

[10] S. Lacey, I.M. White, Y. Sun, S.I. Shopova, J.M. Cupps, P. Zhang, X. Fan, Versatile opto-fluidic ring resonator lasers with ultra-low threshold, Opt. Express 15 (2007) 15523-15530.

[11] J.D. Suter, W. Lee, D.J. Howard, E. Hoppmann, I.M. White, a.X. Fan, Demonstration of the coupling of optofluidic ring resonator lasers with liquid waveguides, Opt. Lett. 35 (2010) 2997-2999.

[12] W. Lee, H. Li, J.D. Suter, K. Reddy, Y. Sun, X. Fan, Tunable single mode lasing from an on-chip optofluidic ring resonator laser, Appl. Phys. Lett. 98(6) (2011) 061103.

[13] H. Azzouz, L. Alkhafadiji, S. Balslev, J. Johansson, N.A. Mortensen, S. Nilsson, A. Kristensen, Levitated droplet dye laser, Optics express 14(10) (2006) 4374-9. 
[14] S.K. Tang, Z. Li, A.R. Abate, J.J. Agresti, D.A. Weitz, D. Psaltis, G.M. Whitesides, A multi-color fast-switching microfluidic droplet dye laser, Lab on a chip 9(19) (2009) 2767-71.

[15] Y. Yang, A.Q. Liu, L. Lei, L.K. Chin, C.D. Ohl, Q.J. Wang, H.S. Yoon, A tunable 3D optofluidic waveguide dye laser via two centrifugal Dean flow streams, Lab on a chip 11(18) (2011) 3182-7.

[16] M.C. Gather, S.H. Yun, Single-cell biological lasers, Nature Photon. 5(7) (2011) 406-410.

[17] R. St-Gelais, J. Masson, Y.A. Peter, All-silicon integrated Fabry-Pérot cavity for volume refractive index measurement in microfluidic systems, Appl. Phys. Lett. 94(24) (2009) 243905.

[18] Y. Guo, H. Li, K. Reddy, H.S. Shelar, V.R. Nittoor, X. Fan, Optofluidic Fabry-Pérot cavity biosensor with integrated flow-through micro-/nanochannels, Appl. Phys. Lett. 98(4) (2011) 041104.

[19] M. Trupke, E.A. Hinds, S. Eriksson, E.A. Curtis, Z. Moktadir, E. Kukharenka, M. Kraft, Microfabricated high-finesse optical cavity with open access and small volume, Appl. Phys. Lett. 87(21) (2005) 211106.

[20] Y.S. Ow, M.B.H. Breese, S. Azimi, Fabrication of concave silicon micro-mirrors, Opt. Express 18(14) (2010) 14511-14518.

[21] M. Malak, N. Gaber, F. Marty, N. Pavy, E. Richalot, T. Bourouina, Analysis of Fabry-Perot optical micro-cavities based on coating-free all-Silicon cylindrical Bragg reflectors, Opt. Express 21(2) (2013) 2378-2392.

[22] D. Hunger, T. Steinmetz, Y. Colombe, C. Deutsch, T.W. Hänsch, J. Reichel, A fiber Fabry-Perot cavity with high finesse, New J. Phys. 12(6) (2010) 065038.

[23] A. Muller, E.B. Flagg, J.R. Lawall, G.S. Solomon, Ultrahigh-finesse, low-mode-volume Fabry-Perot cavity, Opt. Lett. 35(13) (2010) 2293-2295.

[24] D. Hunger, C. Deutsch, R.J. Barbour, R.J. Warburton, J. Reichel, Laser micro-fabrication of concave, low-roughness features in silica, AIP Advances 2(1) (2012) 012119.

[25] O. Svelto, Principles of Lasers, Springers2010. 